\title{
Microstructural Studies of Materials DD1 and KT2 By X-Ray Diffraction
}

\author{
Lakel A*
}

\begin{abstract}
Laboratoire TTSM, Université Biskra, Algérie.
* Corresponding Author: lakel06@yahoo.fr
\end{abstract}

Received: 14-08-2018

Accepted: 28-02-2019

\begin{abstract}
In this work, we have studied two Kaolin .kaolin DD1 which consists of two main phases (kaolinite and Halloysite) and Tamazert kaolin .kaolin KT2 whose main constituents are kaolinite, quartz and mica. Our choice was set on the component that is Kaolinite because of the existence of the latter in both kaolins. A correction of Lorentz-Polarization was carried out prior to the diffraction data, which has been achieved using LWL program dome. The true profile was extracted by this program. The methods used for the micro structural analysis of the present in the two Kaolins are the Warren - Averbach and the Williamson Hall methods Scherrer's relationship has been applied in cases where the compound is devoid of strains.

The study revealed that the kaolin Kaolinite DD1 is devoid of micro constraints. Which similar analysis has shown that the Kaolinite in KT2 incorporates the strains. This was confirmed by the Williamson - Hall method as well as Fourier analysis. The evaluation of strains in the Kaolinite of KT2 has been dome the method of Warren - Averbach and Williamson - Hall diagram. The average value of this constraint found by the first method us 0.15 and 0.21 by the second method gave. The average size of crystallites of the Kaolinite in DD1 was found between $29 \AA$ and $230 \AA$.

by the method of Warren - Averbach and about $118 \AA$ A by the method of Williamson - Hall. Range the second Kaolinite the Kaolinite KT2, the size obtained was respectively $98 \AA$ and $130 \AA$ A using to by methods. The study of the size distribution showed that the dominant size of Kaolinite in DD1 and KT2 is about $40 \AA \AA$ (42\%) and $58 \AA$ (32\%). respectively.
\end{abstract}

Key words: DRX, LWL methods, Crystallites size, microdéfomation, Williamson-Hall and Warren \& Averbach methods.

\section{Introduction}

The microstructural parameters of a material (sizes, shape of the crystallites, external and internal stresses in the crystals) are generally obtained by first extracting the true profile of the sample using a deconvolution operation of Profiles of diffraction lines for powdered compounds. For a microstructural study, the methods of Rent, Weigel and Louboutin (LWL) and that of Stokes are used in the deconvolution of the profiles of diffraction lines. These techniques are said to be rigorous because they do not make assumptions about the nature of the diffraction line profiles. Nevertheless, they use the Fourier coefficients to approximate the function of the 'true' profile of the diffraction peak. Consequently, they are very sensitive to the number of these coefficients which implies the truncation effect (low number of coefficients) but also the cumulative error effect (large number of coefficients). For this reason, it is interesting to take into account the number of Fourier coefficients concerning the X-ray diffraction peaks. The method best suited to this study, where there is a possibility of the presence of a double sizestress effect, is Warren and Averbach's method. The latter will be used in conjunction with the Williamson-Hall method (Balzar, 1999; Kamminga \& Seijbel, 2004; Uvarov \& Popov, 2007; Marinkovic et al., 2001; Larson \& Von Dreele, 2000; Vives et al., 2002; Williamson \& Hall, 1953; Vives et al., 2004; Rehani et al., 2006; Mote, et al., 2012; Langford et al., 1992; Langford , 1978; Wagner, 1966; Rao \& Houska, 1986; Klug \& Alexander, 1974). 
Kaolins are part of the family of clays with montmorillonites, vermiculites, palygostites, The main phase constituting the kaolin is kaolinite or l'Halloysite. Often it is the older rocks, originally rich in feldspar, which decompose under the influence of reducing agents such as carbon dioxide, in kaolins and clays. All kaolin, formed by primary deposit contains beside hydrosilicate of alumina, kaolinite or halloysite, debris important rock undecomposed residue and non removed from the decomposition of which are essentially free quartz mica, magnetite, titanium minerals and other impurities hardly destructible. Quality of kaolin depends on many other compounds that it contains kaolinite as well as the nature and concentration of impurities. Often crude kaolins are directly usable in that state (in the case of kaolin-Djebbel Debbagh, eastern Algeria), but they can also be enriched by kaolinite removal of all or part of the grading methods (case of kaolin tamazert, East Algeria).

\section{Methods of Analysis}

There are several methods to determine the microstructural parameters from the analysis of the peaks of X-ray diffraction. The methods based on the analysis of a single diffraction peak are constructed on fairly restrictive assumptions about the shape of the peaks and the contribution of the effects of size and shape. The Warren-Averbach method requires no assumption about the shape of the diffraction peaks, and it remains the most rigorous. However, it is very sensitive to the phenomenon of truncation profile and an overestimation of background noise. This method can lead to an error of $20-30 \%$ on the determination of the Fourier coefficients and hence the microstructural parameters (Ceretti, 2004). We have used this method in addition to one that is the Williamson-Hall (Vives et al., 2006), which is used in case of presence of microstrain in the crystallites.

\subsection{Methods of evaluation of the size}

To calculate the average size, there are several methods in case the absence of micro strains in the sample.

\subsubsection{Relation of Scherrer}

Scherrer hasshownthatcuts her cristallites in the sample (when the latter does not contain of forced micro) vary in the opposite direction of the width of ray of diffraction. The relation enters those two sizes is given by (Balzar, 1999):

$D^{f}=\mathrm{C} \lambda / \beta \cos \theta$

For widths specified in $2 \theta ; C$ is a coefficient which depends on the shape of the crystallites (Langford et al., 1992) and can assume values between 0.8 and 1.39. For spherical particles, it is generally equal to 1 .

$D^{f}=1 / \beta^{*}$ For widths in units where reciprocal $\theta$ is the Bragg angle, $D^{\mathrm{f}}$ is the average grain size, $\lambda$ is the wavelength and $\beta^{*}$ is the integral width characterizing enlargement, expressed as the reciprocal lattice.

\subsubsection{Diagram Williamson - Hall}

This method applies to types of voightian or pseudo-voget profiles. It uses what is commonly called the Willamson \& Hall graph (Williamson \& Hall, 1953). This graph, to be a straight line, gives valuable information on the presence or absence of microstrain in the sample. The slope of the line indicates the presence of the latter (see Figure 1).

If the profiles are purely Gaussian or purely voget, this method provides a very simple and fast way to evaluate the effects of constraint and size. For the other pseudo-voighal profiles, 
approximations are necessary for the separation of these two effects (Langford et al., 1992; Langford, 1978; Wagner, 1966).

$\beta^{*}=D^{f}+d^{*}-D^{f}$ is the average grain size. $D^{\prime}$ or: $D^{f}=\beta^{*}-d^{*}$

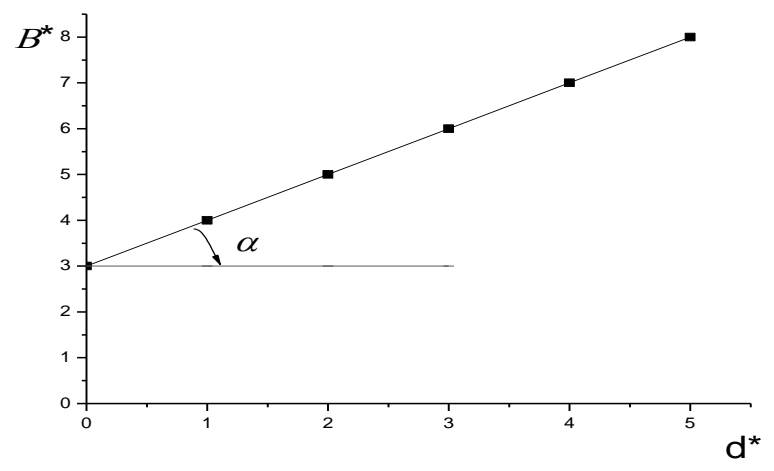

Fig. 1. Diagram of shape Williamson \& Hall.

\subsubsection{Method of Warren - Averbach}

If the sample does not contain microstrain was: $A^{\mathrm{D}}(l, \mathrm{~s})=0$, therefore $A\left(l, s_{n}\right)=A^{s}(l)=$. The initial slope of the curve giving $A\left(l, s_{n}\right)$ according to the length $L$ columns is inversely proportional to the apparent size of the crystallites in a direction perpendicular to the plane $(h k l)$ considered (see Figure 2). This apparent size is given by the following equation (Uvarov \& Popov, 2007):

$$
D_{f}=\left|\frac{d}{A\left(l, s_{n}\right)}\right|_{L \rightarrow 0}, \text { or } L=l^{\Delta s} \text { with: }
$$

$\Delta \mathrm{s}=\lambda_{2}\left(\sin \theta_{2}-\sin \theta_{1}\right) \theta_{1}$ et $\theta_{2}$ are the values of the limit angles that define the profile. From the figure below, the point of intersection of the initial slope of the curve with the x-axis $(L)$ provides the size $D f$.

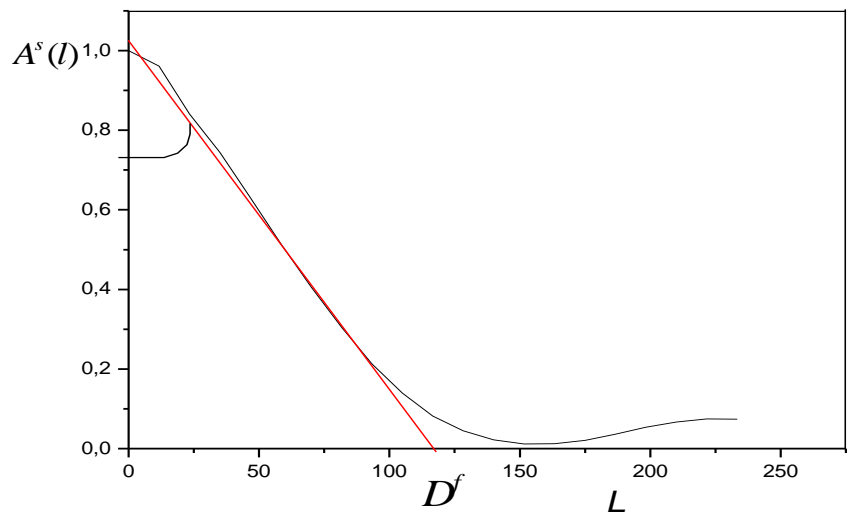

Fig. 2. Fourier Coefficients of As(I) as function of $L$.

\subsection{Measurement Method constraints}

\subsubsection{Method Williamson - Hall}

In the case of the presence of micro-stress, we use the following equation (Williamson-Hall), which allows to obtain the average crystallite size and an estimate of micro-stress (Marinkovic et al., 2001): $\beta(\cos \theta / \lambda)=\left(1 / D^{f}\right)+e_{l}(\sin \theta / \lambda) e_{l}$ is the value associated with the constraint. 
The diagram corresponding to this relationship (giving $\beta{ }^{*}$ depending $\mathrm{d}^{*}$ ) is a straight line whose slope represents the average value of micro-constraints and whose intersection with the horizontal axis gives the average crystallite size.

\subsubsection{Method of Warren - Averbach}

The most valuable for the separation of the size effect and the effect of stress is that of Warren Averbach. Recall that this method is based on the use of data in the form not of a single line but several lines corresponding to different levels of reflection of a family of plans. Profile data are expressed in terms of Fourier coefficients of real A (l, s) and the relationship between the average values of micro-stress and coefficient (Larson \& Von Dreele, 2000):

$$
\begin{aligned}
& \ln \mathrm{A}(l, s)=\ln A^{s}(l)-2 \pi^{2} l^{2} s^{2}\left\langle e_{l}^{2}\right\rangle \\
& \text { i.e.: }\left\langle e_{l}^{2}\right\rangle=\frac{\ln A\left(l, s_{2}\right)-\ln A\left(l, s_{1}\right)}{2 \pi^{2}\left(s_{1}-s_{2}\right)^{2} l^{2}}
\end{aligned}
$$

The coefficients $A^{s}(1)$ which are obtained from this relationship, let be the average size of cristallites.la size $\mathrm{s}$ is equal to the reciprocal variable.

We performed X-ray diffraction recordings on our kaolins Advanced D8 diffractometer. For this, we follow the following steps. Grinding the powder was done to make homogeneous grains. The powder is then deposited on the sample holder and pressed by a glass slide planar surface without too much pressure on the powder in order to avoid preferential orientations. It should be noted that the sample preparation is a critical parameter for obtaining quality results because the main information obtained from diffraction data are influenced by the sample. This information is: the position of the lines, the intensity of the lines, the line shape, the continuous background.

Once the powder deposited on the sample holder, it remains to start recording on the diffractometer. Data recording is carried out on a predefined angular range with a pitch of 0.02 (20) and a counting time by less than three seconds. Qualitative analysis by X-ray diffraction allowed from diffracting planes, highlighting the presence of kaolin DD1 main crystal phases as follows:

Table 1. diffraction planes of the two main phases of DD1.

\begin{tabular}{|c|c|c|}
\hline phase & (hkl) & $2 \theta$ \\
\hline \hline L'halloysite & $(010)$ & 12.36 \\
& $(020)$ & 29.28 \\
\hline La kaolinite & $(200)$ & 19.90 \\
& $(300)$ & 35.02 \\
& $(011)$ & 36.06 \\
& $(201)$ & 38.54 \\
\hline
\end{tabular}

The same way as kaolin DD1, qualitative analysis by X-ray diffraction has highlighted the presence in KT2 kaolin, crystalline phases following Table 2.

Table 2. Plans diffraction phases present in KT2

\begin{tabular}{|c|c|c|}
\hline Phase & $(\mathrm{hkl})$ & $2 \theta$ \\
\hline kaolinite & $(100)$ & 12.40 \\
& $(010)$ & 19.88 \\
& $(200)$ & 24.94 \\
& $(001)$ & 38.52 \\
\hline Quartz & $(201)$ & 20.88 \\
\hline Quartz+Mica & $(101)$ & 26.66 \\
\hline Mica+kaolinite & $(011)$ & 35.00 \\
\hline
\end{tabular}




\section{Results and discussion}

Application of the correction a few peaks our kaolin to see the effect of the application of Lorentz-polarization correction, we chose to make the correction on a dozen peaks kaolins DD1 and KT2. We noticed that the peaks do not change significantly after correction for Lorentz and polarization except for the first peak of each diffractogram which shows differences in a small part at the beginning of the peak. Figure 3 shows the first peak (peak (010)), where we see the difference at the beginning of the peak and another peak (peak (100)) where we see the good superposition of the peak corrected and uncorrected peak.

Similarly, the same figure shows the first peak of the diffractogram KT2 (peak (100)) where the difference is visible in the early peak and a second peak (peak (020) where we see the significant effect of the correction Lorentz-polarization.

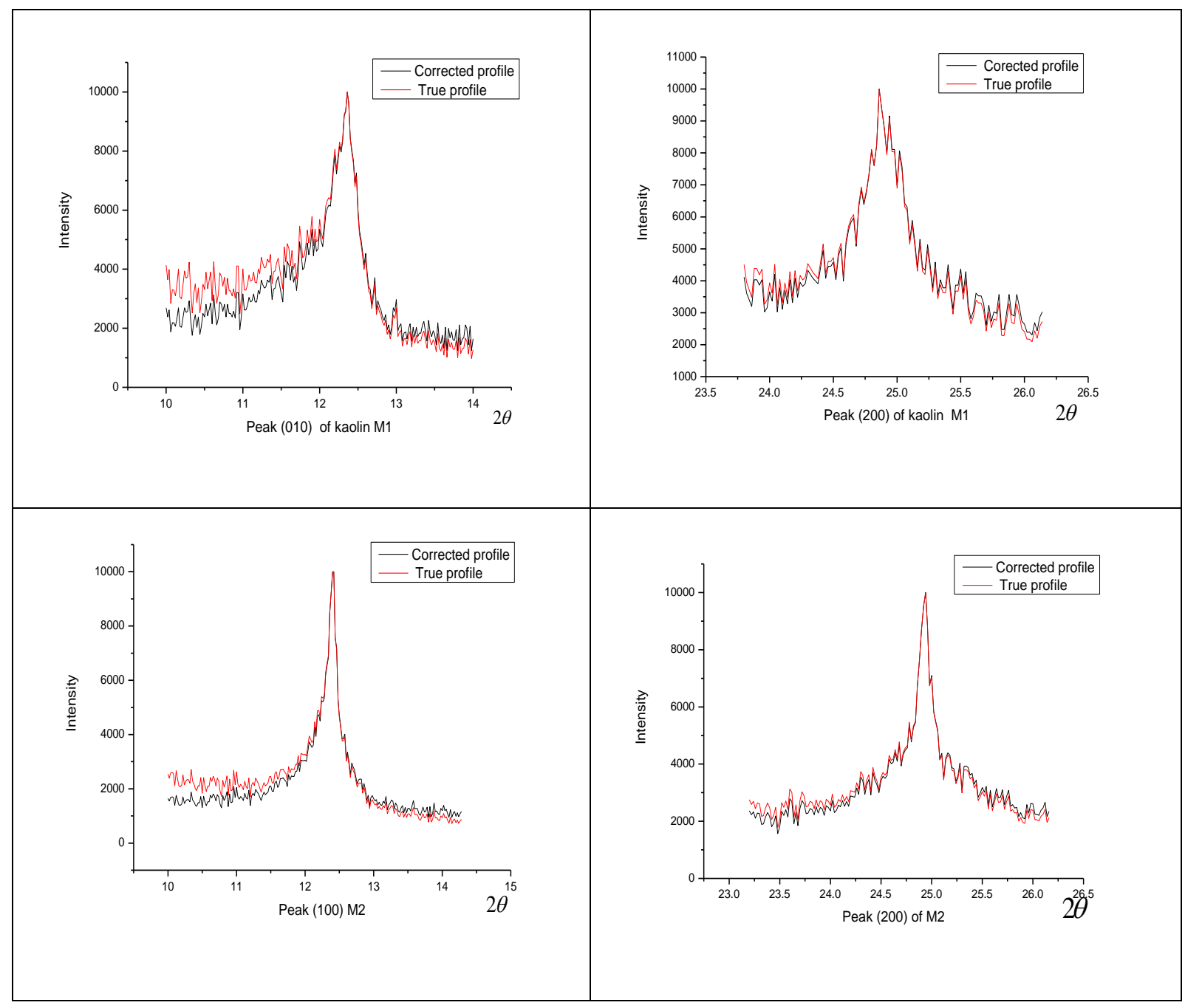

Fig. 3. Effect of correction Lorentz-polarization peaks of some kaolins study corrects red black peak peak uncorrected.

\subsection{Diagram Williamson - Hall}

The Williamson-Hall diagram of Kaolins M1 is given in Figure 4. We represent the values of $\beta^{*}=\beta \cdot \cos \theta / \lambda$ as a function of $d^{*}=\sin \theta / \lambda$. Note that the curve is a straight line and the slope has a value that is negative. The negative value of this slope is probable due to the accuracy 
of the data (obtaining the diffraction data, fitting, removal of the last point in the figure, ...). We therefore deduce that there are no substantial stresses in the kaolinite crystals.

Figure 5 shows the Williamson-Hall diagram of Kaolin M2. Note that the curve is a non-zero slope line. So there are micro-constraints in the sample.

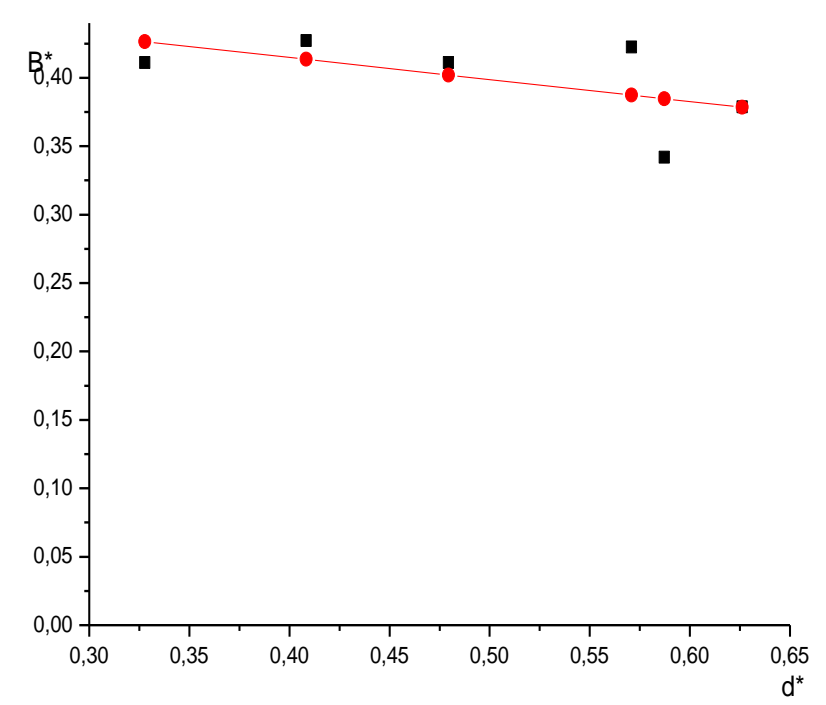

Fig. 4. Wialliamson-Hall diagram of kaolinDD1.

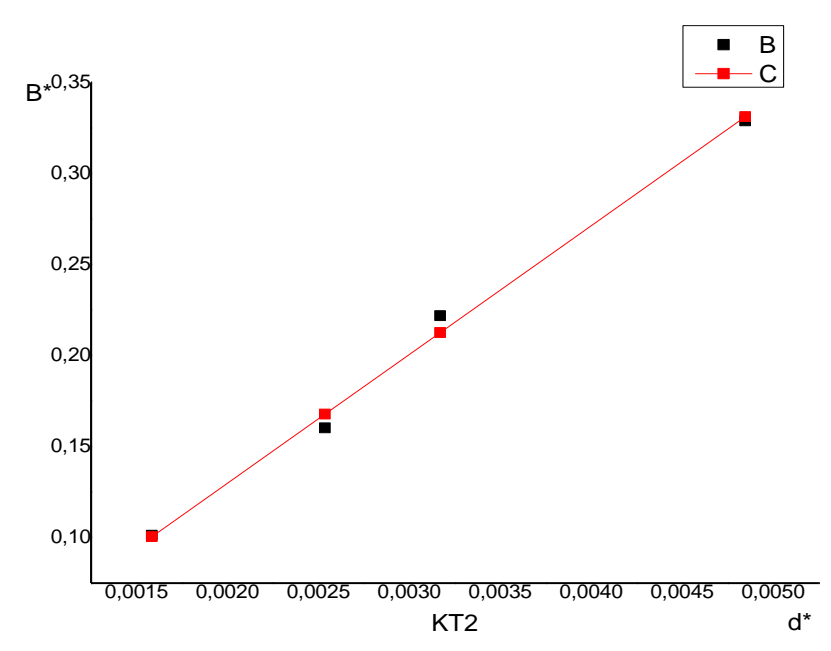

Fig. 5. Diagram of Williamson-Hall of kaolin KT2.

\subsection{Evaluation of the microstrain of kaolin KT2 brut}

The relationships used in the Warren \& Averbach method are:

$$
\begin{aligned}
& \ln A^{D}(l, s)=-2 \pi^{2} s^{2} l^{2}\left\langle e_{l}^{2}\right\rangle \\
& \ln \mathrm{A}(l, s)=\ln A^{s}(l)-2 \pi^{2} l^{2} s^{2}\left\langle e_{l}{ }^{2}\right\rangle
\end{aligned}
$$

These relations can be used with harmonic lines. In our case, we have only two harmonics, they are: (001) and (002). We apply the method to the profiles of these two lines: 
- For the peak (001) et $l=1$ :

$\ln A\left(1, s_{(001)}\right)=\ln A^{s}(1)-2 \pi^{2} s_{(001)}{ }^{2}\left\langle e_{1}^{2}\right\rangle$ ( I )

- For the peak (002) et $l=1$ :

$\ln A\left(1, s_{(002)}\right)=\ln A^{s}(1)-2 \pi^{2} s_{(002)}{ }^{2}\left\langle e_{1}^{2}\right\rangle$

The two previous relationships give the mean values of the size of the crystallites and of the micro-strain present in the sample:

$\left\langle e_{l}{ }^{2}\right\rangle=\frac{\ln A\left(1, s_{(002)}\right)-\ln A\left(1, s_{(001)}\right)}{2 \pi^{2}\left(s_{(001)}{ }^{2}-s_{(002)}{ }^{2}\right)}$

The estimated values for these strains are presented in Table 3. The mean of the set is $\left\langle e_{1}\right\rangle=$ 0.15 .

Table 3. : Evaluation of the stresses exerted on the diffraction planes (hkl).

\begin{tabular}{|c|c|}
\hline $\mathrm{l}$ & $\mathrm{e}_{\mathrm{l}}$ \\
\hline \hline 1 & 0,50 \\
\hline 2 & 0,87 \\
\hline 3 & 1,49 \\
\hline 4 & 1,00 \\
\hline 5 & 0,79 \\
\hline 6 & 0,60 \\
\hline 7 & 0,51 \\
\hline 8 & 0,39 \\
\hline 9 & 0,37 \\
\hline 10 & 0,32 \\
\hline 11 & 0,34 \\
\hline 12 & 0,40 \\
\hline 13 & 0,32 \\
\hline 14 & 0,32 \\
\hline 15 & 0,26 \\
\hline 16 & 0,22 \\
\hline 17 & 0,21 \\
\hline 18 & 0,23 \\
\hline 19 & 0,14 \\
\hline 20 & 0,24 \\
\hline 21 & 0,13 \\
\hline 22 & 0,11 \\
\hline 23 & 0,09 \\
\hline 24 & 0,17 \\
\hline 25 & 0,02 \\
\hline 26 & 0,06 \\
\hline 27 & 0,01 \\
\hline 28 & 0,05 \\
\hline 29 & 0,07 \\
\hline 30 & 0,05 \\
\hline 31 & 0,04 \\
\hline 32 & 0,08 \\
\hline 33 & 0,06 \\
\hline 34 & 0,07 \\
\hline 35 & 0,07 \\
\hline 36 & 0,06 \\
\hline 37 & 0,03 \\
\hline 39 & 0,06 \\
\hline & 0,10 \\
\hline 25,05 \\
\hline 30.15 \\
\hline
\end{tabular}


The Williamson-Hall diagram, shown in Figure 4, provides the estimated value for these constraints : <el $>=0.21$.

\subsection{Evaluation of size in Kaolin KT2 brut}

From the relation 1 of Warren and Averbach, we find the values of the component $A^{\mathrm{s}}(I)$ of $A(1$, $S$ ). The values of these components are written in Table 4.

Table 4. The value of the $A^{s}$ component(I).

\begin{tabular}{|c|c|}
\hline $\mathrm{L}$ & $\boldsymbol{A}^{\boldsymbol{x}}(\boldsymbol{l})$ \\
\hline \hline 1 & 30062 \\
\hline 2 & 113961 \\
\hline 3 & 211566 \\
\hline 4 & 34602 \\
\hline 5 & 45809 \\
\hline 6 & 521 \\
\hline 7 & 61602 \\
\hline 8 & 77992 \\
\hline 9 & 86551 \\
\hline 10 & 980 \\
\hline 11 & 103 \\
\hline 12 & 1153 \\
\hline 13 & 12161 \\
\hline 14 & 1360 \\
\hline 15 & 141611 \\
\hline 16 & 15181 \\
\hline 17 & 16302 \\
\hline 18 & 17747 \\
\hline 19 & 181 \\
\hline 20 & 19587 \\
\hline 21 & 20776 \\
\hline 22 & 21993 \\
\hline 23 & 225 \\
\hline 24 & 2391 \\
\hline 25 & 24681 \\
\hline 26 & 251246 \\
\hline 27 & 26751 \\
\hline 28 & 27363 \\
\hline 29 & 28499 \\
\hline 30 & 29803 \\
\hline 31 & 30133 \\
\hline 32 & 31302 \\
\hline 33 & 3259 \\
\hline 34 & 3322 \\
\hline 35 & 3486 \\
\hline 36 & 35361 \\
\hline 37 & 3683 \\
\hline 38 & 371 \\
\hline 39 & 3892 \\
\hline & \\
\hline 5
\end{tabular}




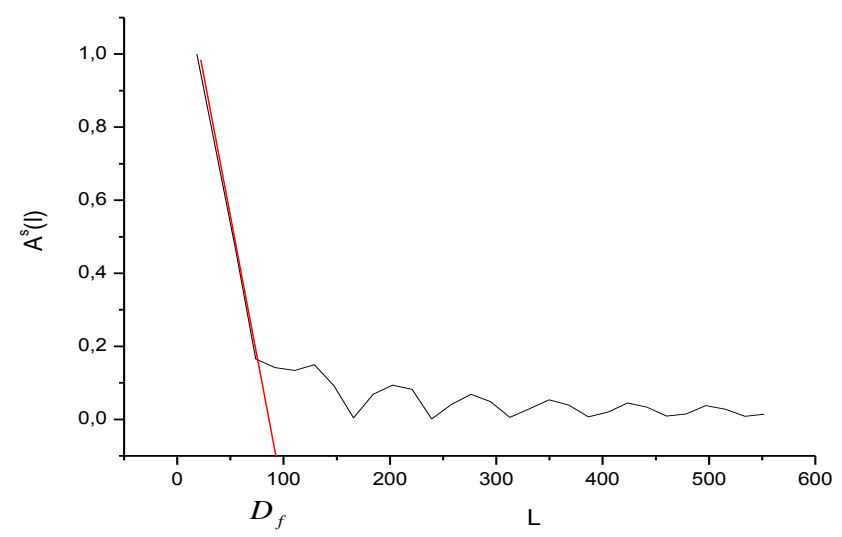

Fig. 6. Variation of the Fourier real coefficients $A(I, s)$ as a function of $L$

From this graph, we derive the value of the mean size of the crystallites in the kaolin M2. This value is: $\angle D f>=92 \AA$.

Evaluation of crystallite size in Kaolin DD1

The average crystallite size of the kaolinite of M1 was calculated by the Warren \& Averbach method $111 \AA$.

Figure7 represents the variation of the coefficients of Fourier based on $L$ (harmonic number).

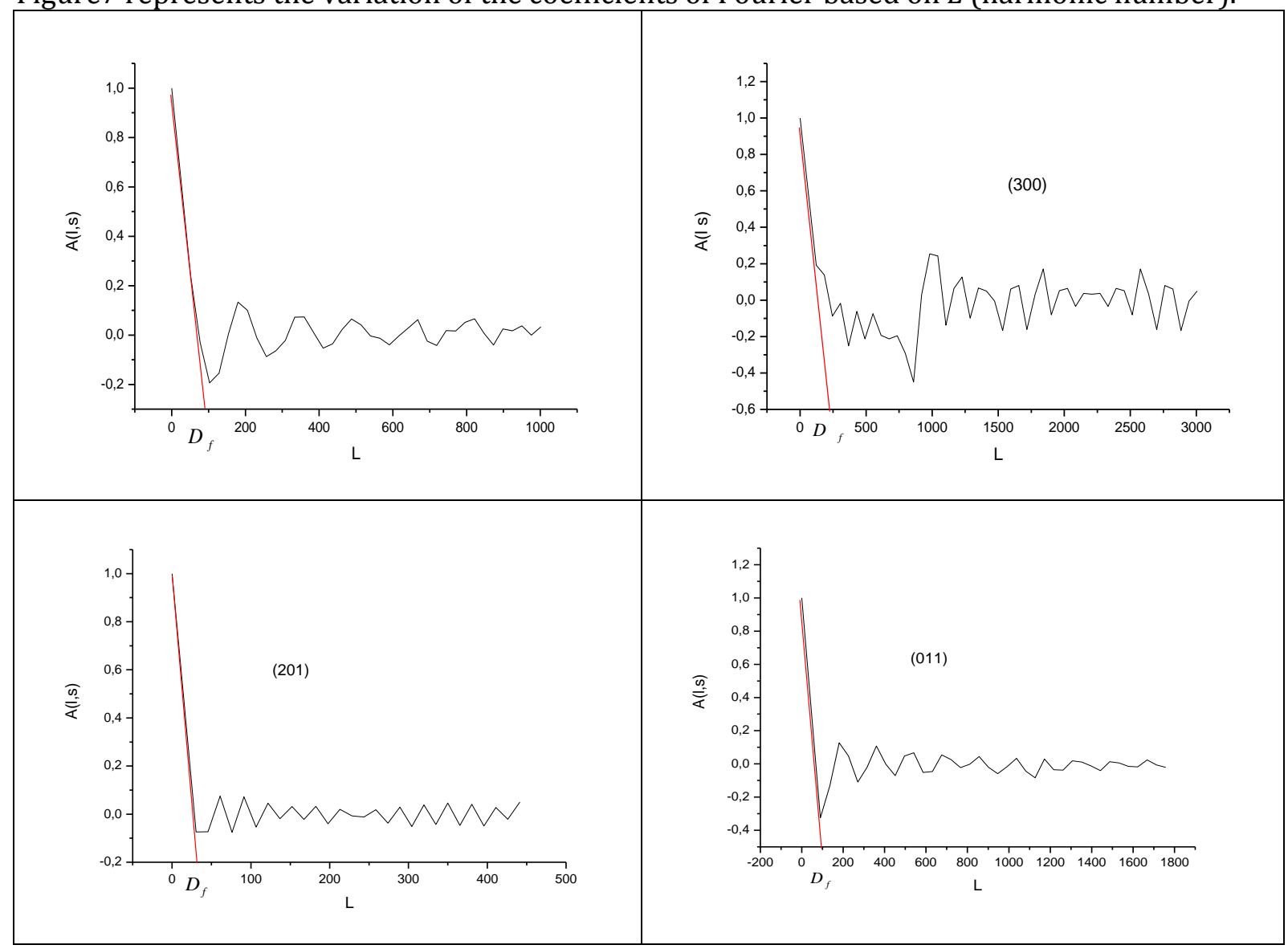

Fig. 7. Variation of the Fourier real coefficients $A(I, s)$ as a function of $L$. 
Table 5. The size of the crystallites of the kaolin DD1brut from the different diffraction peaks.

\begin{tabular}{|c|c|}
\hline The pics & Df $(\AA)$ \\
\hline \hline$(200)$ & 92 \\
\hline$(300)$ & 230 \\
\hline$(011)$ & 94 \\
\hline$(201)$ & 29 \\
\hline \multicolumn{2}{|c|}{ Heightsiz $<\mathrm{D}>=111$} \\
\hline
\end{tabular}

The average crystallite size of the kaolinite of DD1 was calculated by the Scherrer125 Å relation.

Table 6. The size of the crystallites of the kaolin DD1brut from the different diffraction peaks.

\begin{tabular}{|c|c|}
\hline The pics & Df $(\AA)$ \\
\hline$(200)$ & 130 \\
\hline$(300)$ & 125 \\
\hline$(011)$ & 129 \\
\hline$(201)$ & 116 \\
\hline \multicolumn{2}{|c|}{ Height size $<$ Df $>=125 \AA$} \\
\hline
\end{tabular}

From the Williamson-Hall diagram, we derive the average values of crystallite size. The value found for this size is : $D f=118(\AA)$.

As can be seen, the three results obtained from the three methods are sufficiently close:

- By Scherrer ' s relation: $D^{f=125} \AA$.

- By the Williamson - Hall diagram: $D^{f=} 118 \AA$.

- By the method of Warren \& Averbach: $D f=111 \AA$.

\subsection{Size distribution of kaolinite present in DD1}

The size distribution was estimated from the second derivative of the curve $A^{s}(I)$ according to the harmonic number $L$. We used a second degree polynomial for the calculations. We give in the following table only the most common sizes (sizes dominant).

Table 7. Size of the main phase dominant kaolin DD1.

\begin{tabular}{|c|c|}
\hline pics & dominant size $(\AA)$ \\
\hline \hline$(200)$ & 32 \\
\hline$(300)$ & 35 \\
\hline$(011)$ & 40 \\
\hline$(201)$ & 42 \\
\hline
\end{tabular}

\subsection{Size distribution of kaolinite present in KT2 and DD1}

We calculated the size distribution of kaolinite in kaolin DD1. The value found for the dominant size is $40 \mathrm{~A}$. The distribution corresponding to this dominant size is of the order of $42 \%$.

The study of the size distribution of kaolinite in kaolin KT2 gave an average value of $58 \AA$ for the dominant size; it has a distribution of about 32\% (Lakel et al., 2013).

\section{Conclusion}

The methods for the determination of microstructural parameters from the analysis of the diffraction peaks were primarily developed for X-ray diffraction The Warren-Averbach method requires no assumption about the shape of the diffraction peaks and it is the most rigorous. 
However, it is very sensitive to the phenomenon of truncation profile and an overestimation of background noise that can lead to an error of $20-30 \%$ on the determination of the Fourier coefficients.

In the method of Sprauel, the Fourier coefficients are determined by smoothing each diffraction pattern by a mathematical function (generally Voigt), fitted to the experimental values by a least-squares optimization. In addition, this method allows to take into account the effects due to the heterogeneity of elastic deformations. The analysis is performed on two further orders of the same reflection. Methods based on the analysis of a single diffraction peak based on fairly restrictive assumptions about the shape of the peaks and the contribution of the effects of size and shape. They are rather qualitative analysis methods, but quick and easy application. The results obtained in the simplest cases (isotropic cubic) are in agreement with those obtainable with the methods based on the analysis of two orders of reflection.

\section{References}

Balzar, D. (1999). Voigt-function model in diffraction line-broadening analysis. International union of crystallography monographs on crystallography, 10, 94-126.

Ceretti, M. (2004). Apport de la diffraction des neutrons à l'analyse des contraintes internes. Habilitation à diriger des recherches, Université de Paris-Sud, Centre d'Orsay, 7-17.

Kamminga, J. D., \& Seijbel, L. J. (2004). Diffraction line broadening analysis if broadening is caused by both dislocations and limited crystallite size. Journal of research of the National Institute of Standards and Technology, 109(1), 65.

Klug, H. P., \& Alexander, L. E. (1974). X-ray diffraction procedures: for polycrystalline and amorphous materials. X-Ray Diffraction Procedures: For Polycrystalline and Amorphous Materials, 2nd Edition, by Harold P. Klug, Leroy E. Alexander, pp. 992. ISBN 0-471-49369-4. Wiley-VCH, May 1974., 992.

Lakel, A., Bouyoucef, A., Labii, T., Guechi, N., Guechi, I., Boubertakh, A., \& Hamamda, S. (2013). Microstructural Study of Some Kaolin by Warren-Averbach and Williamson-Hall Methods. First International Conference on Renewable Energies and Nanotechnology impact on Medicine and Ecology, ICREN 2013, Algeria-Constantine, february16-17, pp.169-177.

Langford, J. I. (1978). A rapid method for analysing the breadths of diffraction and spectral lines using the Voigt function. Journal of Applied Crystallography, 11(1), 10-14.

Langford, J. I., Prince, E., \& Stalick, J. K. (1992). Accuracy in powder diffraction II. NIST special publication, 846, 110-126.

Larson, A. C., \& Von Dreele, R. B. (2000). Report LAUR 86-748. Los Alamos National Laboratory, New Mexico, USA.

Marinkovic, B., Avillez, R. R. D., Saavedra, A., \& Assunção, F. C. R. (2001). A comparison between the Warren-Averbach method and alternate methods for X-ray diffraction microstructure analysis of polycrystalline specimens. Materials Research, 4(2), 71-76..

Mote, V. D., Purushotham, Y., \& Dole, B. N. (2012). Williamson-Hall analysis in estimation of lattice strain in nanometer-sized ZnO particles. Journal of Theoretical and Applied Physics, 6(1), 6.

Rao, S., \& Houska, C. R. (1986). X-ray particle-size broadening. Acta Crystallographica Section A: Foundations of Crystallography, 42(1), 6-13.

Rehani, B. R., Joshi, P. B., Lad, K. N., \& Pratap, A. (2006). Crystallite size estimation of elemental and composite silver nano-powders using XRD principles, 44, 157-161.

Uvarov, V., \& Popov, I. (2007). Metrological characterization of X-ray diffraction methods for determination of crystallite size in nano-scale materials. Materials characterization, 58(10), 883891.

Vives, S., Gaffet, E., \& Meunier, C. (2004). X-ray diffraction line profile analysis of iron ball milled powders. Materials Science and Engineering: A, 366(2), 229-238. 
Vives, S., Gaffet, E., Itié, J. P., \& Meunier, C. (2002). Influence des conditions de broyage sur la nanostructure et le module de compression de poudres de fer. Matériaux, 1-4

Wagner, C. N. J. (1966). Local atomic arrangements studied by X-ray diffraction. Gordon and Breach, New York, 219.

Williamson, G. K., \& Hall, W. H. (1953). X-ray line broadening from filed aluminium and wolfram. Acta metallurgica, 1(1), 22-31. 